\title{
Chronic Venous Disorders: A Contemporary Review
}

\author{
Abul Hasan Muhammad Bashar \\ Department of Vascular Surgery, NICVD, Dhaka
}

\begin{abstract}
:
Key Words : $\quad$ Chronic venous disease is a common medical condition of the lower limbs that affects a large number Venous disorder, of people in every part of the world including the Indian subcontinent. The disease is insidious in endovascular therapy. nature and therefore often ignored in the early stages. In advanced stages, it causes significant discomfort in the affected limbs which adversely influences quality of life, work hours and treatment expenses. The present article reviews the various aspects of the disease including its pathophysiology and recent management updates.
\end{abstract}

(Cardiovasc. j. 2019; 12(1): 59-63)

Definitions: Chronic Venous Disorders, Disease \& Insufficiency:

Chronic Venous Disorders: Chronic Venous Disorders is an all-inclusive term that encompasses a spectrum of venous diseases from simple telangiectasias (also known as spider veins) and reticular veins, varicose veins, leg edema to more severe form of venous disorders including skin changes and ulcerations (C1-6). Chronic Venous Disease (CVD) is a more commonly used terminology and represents a part of the spectrum of chronic venous disorders which include varicose veins, edema and skin changes and ulceration affecting the lower limb. A third terminology to describe venous disease is Chronic Venous Insufficiency (CVI) which denotes Chronic Venous Disorders with manifestations specific to abnormal venous function. CVI indicates more advanced forms of CVD and includes manifestations such as skin pigmentation, venous eczema, lipodermatosclerosis, atrophie blanche and healed and active ulcers ${ }^{1}$.

\section{Classification of Chronic Venous Disease:} The CEAP classification:

The most widely used classification to categorize CVD is the CEAP (Clinical-Etiologic-AnatomicPathophysiologic) classification. It was developed in 1994 by an international ad hoc committee of the
American Venous Forum. It was endorsed by the Society for Vascular Surgery, and incorporated into "Reporting Standards in Venous Disease" in 1995. The brief outline of the CEAP classification is as follows;

\section{Clinical:}

\begin{tabular}{|c|c|}
\hline $\begin{array}{l}\text { C } 0-\text { no evidence of } \\
\text { venous disease }\end{array}$ & $\begin{array}{l}\text { C } 1 \text { - telangiectasias/ reticular } \\
\text { veins }\end{array}$ \\
\hline $\mathrm{C} 2-$ & $\begin{array}{l}\text { C } 3 \text { - edema associated with } \\
\text { vein disease }\end{array}$ \\
\hline C $4 \mathrm{a}$ - pigmentation & $\begin{array}{l}\text { C } 4 b \text { - lipodermatosclerosis } \\
\text { or eczema }\end{array}$ \\
\hline $\begin{array}{l}\text { C } 5 \text { - healed venous } \\
\text { ulcer (Figure } 1 \mathrm{~A} \text { ) }\end{array}$ & $\begin{array}{l}\text { C } 6 \text { - active venous ulcer } \\
\text { (Figure } 1 \mathrm{~B} \text { ) }\end{array}$ \\
\hline
\end{tabular}

\section{Etiologic:}

Ec: congenital, Ep: primary, Es: secondary (post thrombotic), En: no venous cause identified

\section{Anatomic:}

As: superficial veins, Ap: perforator veins, Ad: deep veins, An: no venous location identified

\section{Pathophysiologic:}

Pr: reflux, Po: obstruction, Pr,o: reflux and obstruction, $\mathrm{Pn}$ : no venous pathophysiology identifiable

Address of Correspondence: Dr. Abul Hasan Muhammad Bashar, Department of Vascular Surgery, National Institute of Cardiovascular Diseases, Dhaka, Bangladesh. Email: ahmbashar@gmail.com

(C) 2018 authors; licensed and published by International Society of Cardiovascular Ultrasound, Bangladesh Chapter and Bangladesh Society of Geriatric Cardiology. This is an Open Access article distributed under the terms of the CC BY NC 4.0 (https://creativecommons.org/licenses/by-nc/4.0). 


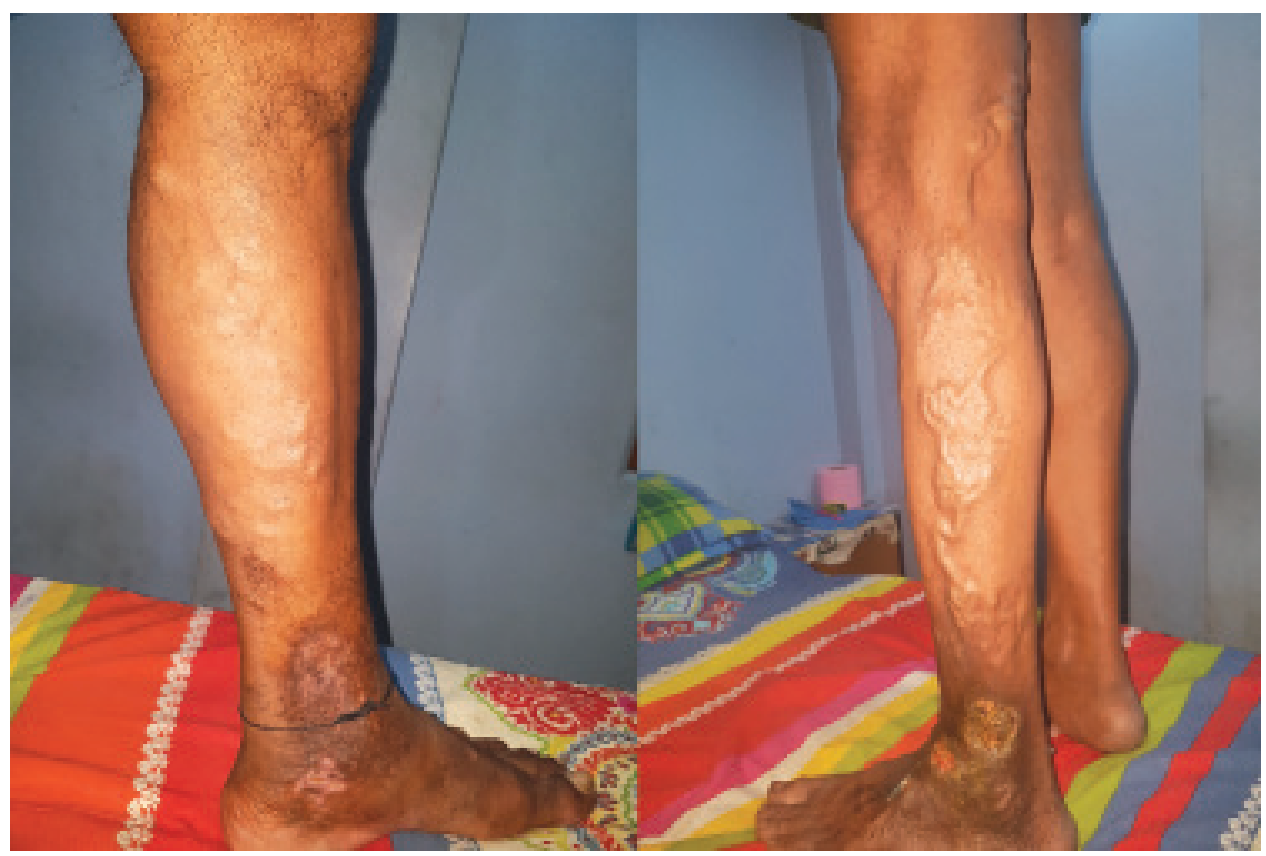

(a)

(b)

Fig 1: CVD presenting in CEAP clinical class C5 and C6.

Though CEAP has undergone revisions to make it more versatile, the basic CEAP remains the most commonly used tool to describe and report venous diseases $^{2}$.

\section{Magnitude of the Problem:}

CVD is a very common problem with varicose veins affecting more than 25 million adults in the United States and more than 6 million with more advanced venous disease. ${ }^{3}$ Because of this high prevalence of venous disease, the National Venous Screening Program was conducted by the American Venous Forum in the United States to increase awareness. The program identified varicose veins in $>30 \%$ of participants and more advanced venous disease in $>10 \% .{ }^{4}$ The vein consult program $(\mathrm{VCP})$ is a prospective survey aiming to collect global epidemiological data on chronic venous disorders (CVD) based on the CEAP classification. It enrolled 6232 general practitioners and 91545 subjects over the age of 18 from all over the world. The worldwide prevalence of CVD was $83.6 \%$; $63.9 \%$ of the subjects ranging $\mathrm{C} 1$ to $\mathrm{C} 6$, and $19.7 \%$ being $\mathrm{C} 0$ s subjects. $\mathrm{C} 0$ s patients were more frequently men whatever the age and the geographical zone. C1-C3 appeared to be more frequent among women whatever the country but the rate of severe stages (C4-C6) did not differ between men and women. ${ }^{5}$ Estimates from the west show that prevalence of varicose veins varies widely from $2-56 \%$ in men and from $1-60 \%$ in women and venous ulceration affect approximately $0.3 \%$ of the adult population. ${ }^{6}$ According to an Indian survey, the prevalence of varicose veins in India is $15-20 \%{ }^{7}$ In Bangladesh, there is no published data regarding the prevalence of CVD. However, unpublished data from the National Institute of cardiovascular diseases (NICVD) suggest that about $50 \%$ of all patients undergoing Duplex study for vascular diseases are venous patients and more than $80 \%$ of venous consultations are for C4-C6 disease.

\section{Pathophysiology of CVD:}

Increased venous pressure or venous hypertension lies at the heart of the pathogenesis of CVD. This may be caused by a number of pathologic conditions such as valvular incompetence in the superficial or deep axial veins, perforator incompetence, venous obstruction etc. A combination of the abovementioned factors may also be present. The pathophysiology leading to venous hypertension is highly complex and multifactorial. Inflammatory cells have a central role in the pathophysiology of chronic venous disease and venous leg ulcers. Endothelial dysfunction and expression of adhesion molecules are also important pathophysiological factors that attract leukocytes initiating events of 
inflammatory response. 8 Cytokines and matrix metalloproteinases are expressed in chronic venous disease. Genetic predisposition, environmental and hormonal factors have also been implicated in the pathogenesis of CVD.

\section{Evaluation of CVD patients:}

Evaluation of CVD cases is based on thorough clinical examination which is supplemented by Vascular Duplex study in most cases. Venous Duplex Imaging done expeditiously in expert hand not only confirms diagnosis, it also gives vital clues to etiology, anatomy and pathophysiology. Computed Tomographic or Magnetic Resonance Venography is occasionally done to evaluate the proximal veins with a view to judge suitability of interventions like venous stenting to improve outflow. Ascending venography is also used in selective cases. Modalities like plethysmography and ambulatory venous pressure measurement are rarely used in Bangladesh.

\section{Treatment of CVD patients:}

The goal of CVD treatment is to reduce venous hypertension by decompressing or terminating the sources of increased venous pressure. To that goal, the following treatment options are in practice;

1. Conservative

2. Sclerotherapy

3. Surgery

4. Endovenous Laser Ablation (EVLA)

5. Radio Frequency Ablation (RFA)

\section{Conservative:}

External compression and flavonoids form the mainstay of the conservative management. Graduated compression stockings (GCS) is recommended for CVD patients of all CEAP classes. It helps to reduce ambulatory venous hypertension and improve microcirculation, filtration and exchange dynamics. Stockings can be of varying pressure. Generally, an external compression pressures of 20 to $30 \mathrm{mmHg}$ at the ankle with decreased proximal compression promotes forward flow. ${ }^{9}$ Avoiding prolonged standing or sitting and reducing body weight also help in reducing venous hypertension.

The role of Micronized Purified Flavonoid Fraction (MPFF) has been well documented in different clinical trials. ${ }^{10}$ Well tolerated, this class of drug has been found efficacious in increasing venous tone, suppress WBC activation and decrease release of inflammatory mediators, decreases capillary hyperpermeability and fragility and increases lymphatic drainage. Thus, they reduce edema, improve skin changes and improve quality of life. MPFF are effective in all clinical classes of CVD and also after stripping and endovenous treatments.

\section{Sclerotherapy:}

In foam sclerotherapy, the dilated superficial veins are cannulated preferably under ultrasound guidance and injection of a foaming agent or sclerosant (for example, Polydocanol, Sodium tetradecyl sulfate etc.). The foam, upon contact with the vessel wall, leads to endothelial denudation, thrombosis, and occlusion with sclerosis of the vein. Sclerotherapy is practiced independently for minor varicosities or can be used in conjunction with surgery/endovenous procedures.

\section{Surgery:}

This is the traditional method of treating primary varicose veins. It usually involves flush ligation of the refluxing vein at their junction with the deep vein (Sapheno-Femoral or Sapheno-Popliteal junction) with or without stripping combined with phlebectomy when necessary (Figure 2).

\section{EVLA/RFA:}

Endovenous techniques such as Radiofrequency and laser ablation are relatively recent additions to treatment options of primary varicose veins. They use thermal energy to damage and occlude the venous wall. Both techniques have been successfully used to treat reflux of the long and short saphenous veins (Figure 3). With ClosureFast ${ }^{\mathrm{TM}}$ Endovenous Radiofrequency Ablation Catheter (Medtronic, Inc.; Minneapolis, Minn), occlusion rates of $100 \%$ at 1 week, $97 \%$ to $99.7 \%$ at 3 months, $95 \%$ to $98 \%$ at 1 year, and $85 \%$ to $93 \%$ at 3 years have been reported. ${ }^{11,12}$ Longer term benefit has also been demonstrated in five-year follow-up data that revealed a shift from CEAP class C2 to class C1 after ablation. Risks and complications of all these procedures include perforation, deep vein thrombosis, pulmonary embolism, phlebitis, hematoma, infection, skin burns, and nerve injury.

A randomized clinical trial by Rasmussen and colleagues ${ }^{13}$ involving 500 patients compared the 


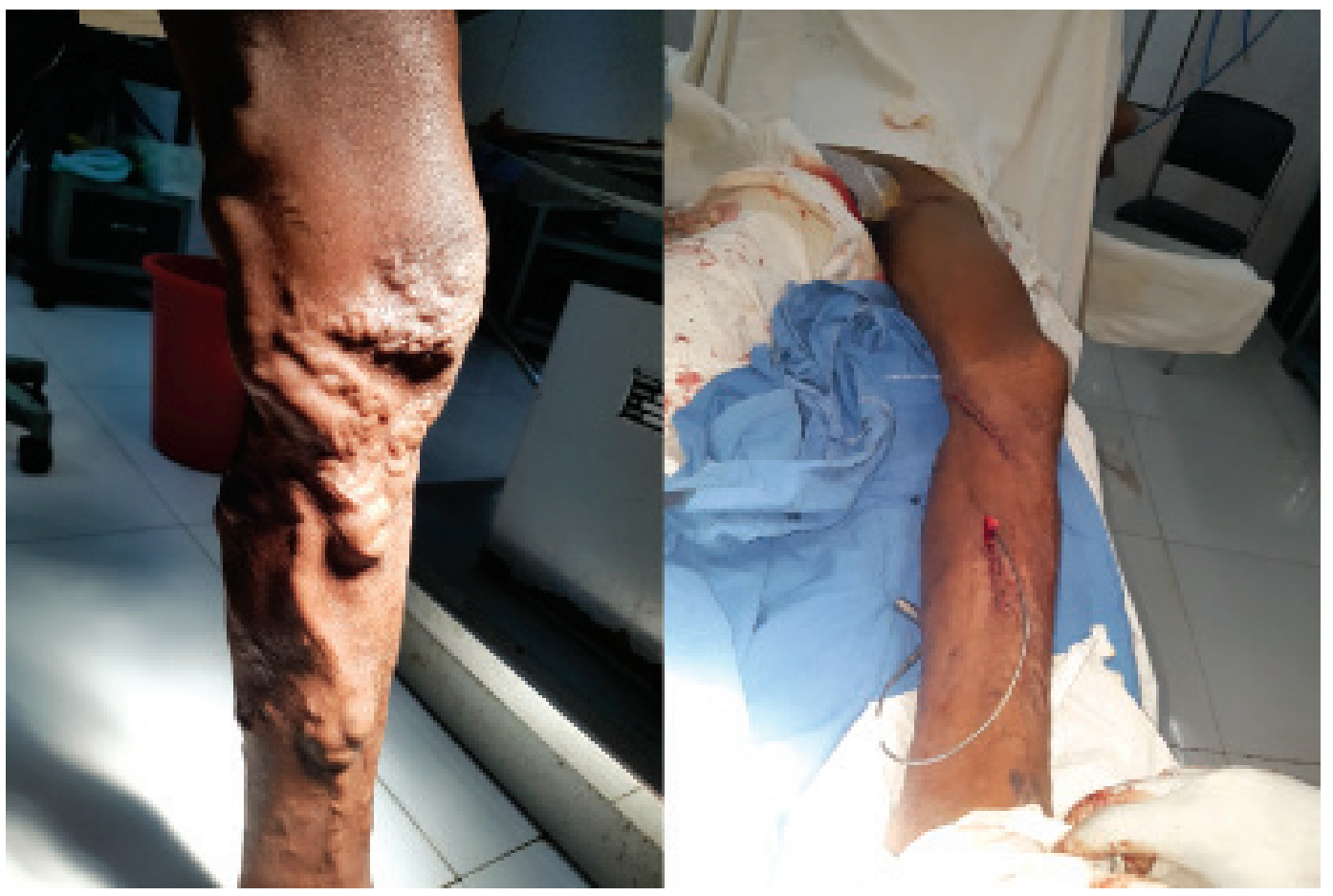

Fig.-2: Surgical treatment of extensive primary varicose vein.

treatment of long saphenous varicosities using the aforementioned methods. The clinical effectiveness of RFA, EVLA and stripping as defined by refluxfree rate at one year was found superior to that of foam sclerotherapy (RFA - 95.2\%, EVLA- 94.2\%, and stripping- $95.2 \%$; foam sclerotherapy$83.7 \% ; P<0.001)$. The time until return to work was significantly shorter after RFA and foam sclerotherapy (both $2.9 \mathrm{~d}$ ) than after EVLA and stripping (3.6 and $4.3 \mathrm{~d}$, respectively; $P<0.001$ ).

\section{Interventional Treatment of CVD:}

Many CVD patients particularly those of post thrombotic syndrome or May-Thurner syndrome (Iliac vein compression by crossing Iliac artery) may benefit from interventional treatment like Percutaneous Transluminal Angioplasty with stenting of the obstructed proximal veins to improve outflow (Figure 4). A relatively recent trend in the treatment of venous diseases of thrombotic or fibrotic etiology, interventional treatment involves

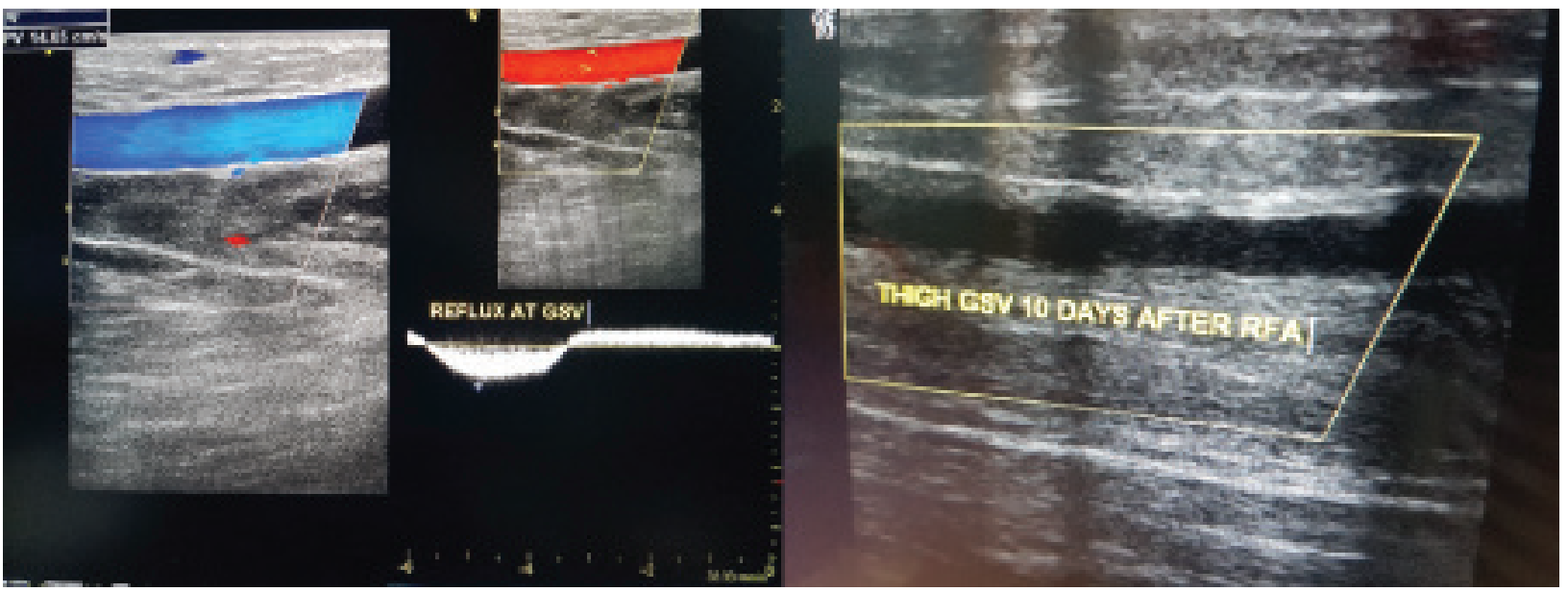

Fig.-3: Radio-Frequency ablation of refluxing great saphenous vein. 


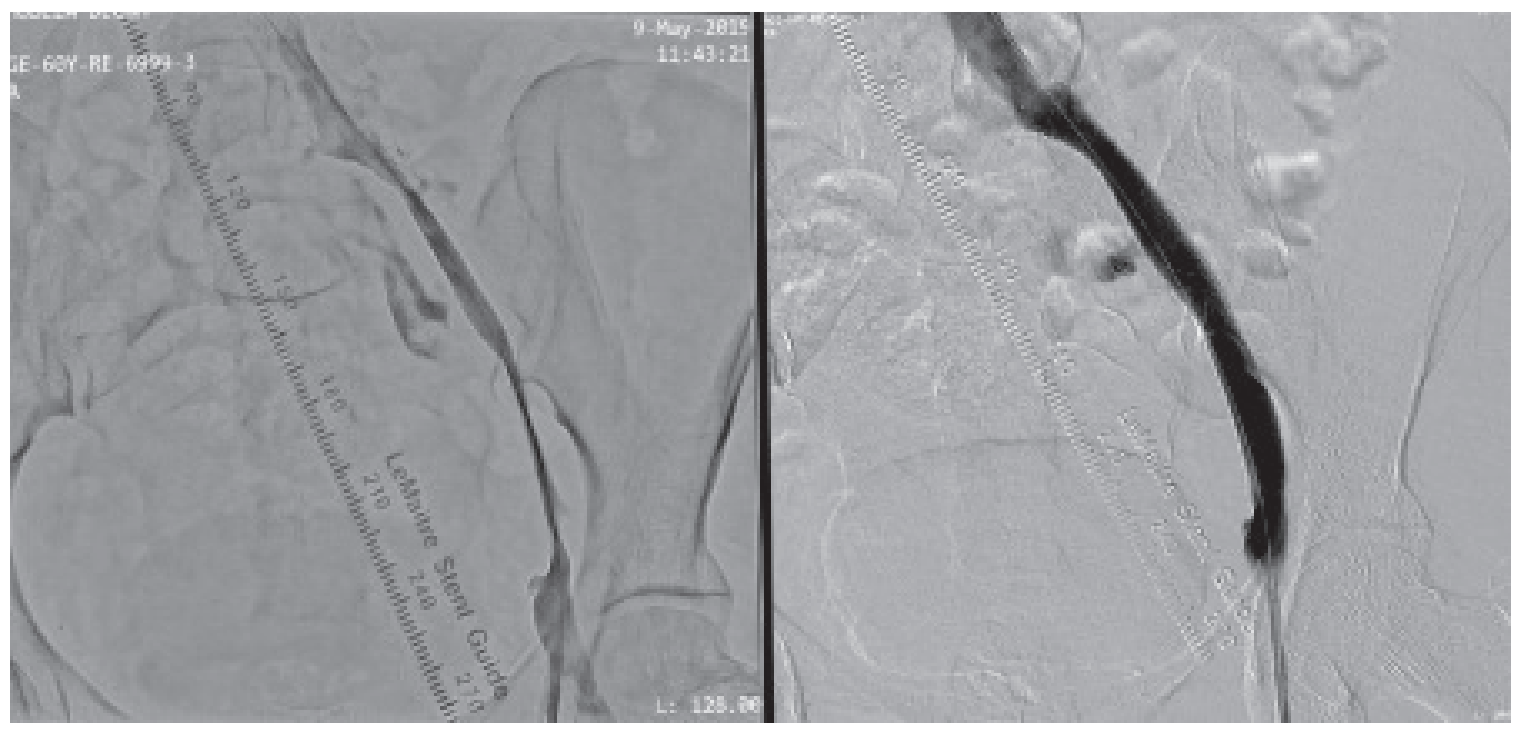

Fig.-4: Stenting for long segment Iliac vein stenosis due to chronic deep vein thrombosis.

considerable resource mobilization which is a major constraint in the perspective of Bangladesh.

\section{Conclusion:}

Statistically, CVD is ten times more prevalent than peripheral arterial disease. ${ }^{9}$ However, due to its insidious nature, it is often ignored by the patients particularly in countries like Bangladesh. Most patients present late with significant morbidity and complications which has considerable socio-economic implications. Generating social awareness about CVD can bring a welcome change in this situation. Treatment of earlier stages of CVD is mostly conservative. Surgery and endovenous procedures which are all available in Bangladesh are reserved for cases presenting in late stages.

\section{Conflict of Interest - None.}

\section{References:}

1. Reffetto JD, Eberhardt RT. Chronic Venous Disorders: General Considerations. In Cronenwett JL, Johnston KW. Eds. Rutherford's Vascular Surgery. $7^{\text {th }}$ edition. Philadelphia: Elsevier Saunders, 2010: 831-843.

2. Eklöf B, Rutherford RB, Bergan JJ, Carpentier PH, Gloviczki P, Kistner RL, et al. Revision of the CEAP classification for chronic venous disorders: Consensus statement. J. Vasc Surg 2004; 40 (6):1248-1252. DOI: 10.1016/j.jvs.2004.09.027

3. Beebe-Dimmer JL, Pfeifer JR, Engle JS, Schottenfeld D. The epidemiology of chronic venous insufficiency and varicose veins. Ann Epidemiol 2005; 15: 175-184.

4. McLafferty RB, Passman MA, Caprini JA, Rooke TW, Markwell SA, Lohr JM, et al. Increasing awareness about venous disease: the American Venous Forum expands the National Venous Screening Program. $J$ Vasc Surg 2008; 48: 394-399.
5. Rabe E, Guex JJ, Puskas A, Scuderi A, Fernandez Quesada F. Epidemiology of chronic venous disorders in geographically diverse populations: results from the Vein Consult Program. Int Angiol 2012; 31: 105-115.

6. Robertson L, Evans C, Fowkes FG. Epidemiology of chronic venous disease. Phlebology 2008;23(3):103-111.

7. Pradeep Muley. Treating varicose veins without surgery. Available from: http:// www. indianinterventionalradiology.in. Accessed on May 03 2008.

8. Raffetto JD. Pathophysiology of Chronic Venous Disease and Venous Ulcers. Surg Clin North Am 2018; 98(2): 337-347.

9. Michael McArdle, Eduardo A. HernandezVila. Management of Chronic Venous Disease. Tex Heart Inst J 2017 Oct; 44(5): 347-349. doi: 10.14503/ THIJ-17-6357

10. Kakkos SK, Nicolaides AN. Efficacy of micronized purified flavonoid fraction (Daflon ${ }^{\circledR}$ ) on improving individual symptoms, signs and quality of life in patients with chronic venous disease: a systematic review and meta-analysis of randomized double-blind placebocontrolled trials. Int Angiol 2018 Apr; 37(2): 143-154. doi: 10.23736/S0392-9590.18.03975-5

11. Braithwaite SA, Braithwaite BD. Clinical utility of the Covidien Closure Fast endovenous radiofrequency ablation catheter. Med Devices (Auckl) 2014; 7: 179185. doi: 10.2147/MDER.S48141

12. Goodyear SJ, Nyamekye IK. Radiofrequency ablation of varicose veins: best practice techniques and evidence. Phlebology 2015;30(2 Suppl):9-17. doi: 10.1177/ 0268355515592771

13. Rasmussen LH, Lawaetz M, Bjoern L, Vennits B, Blemings A, Eklof B. Randomized clinical trial comparing endovenous laser ablation, radiofrequency ablation, foam sclerotherapy and surgical stripping for great saphenous varicose veins. Br J Surg 2011;98(8):10791087. doi: 10.1002/bjs. 7555 